\title{
Thermographic determination of the sheath heat transmission coefficient in a high density plasma
}

\author{
M.A. van den Berg ${ }^{\mathrm{a}^{*}}$, K.Bystrov ${ }^{\mathrm{a}}$, R. Pasquet ${ }^{\mathrm{a}}$, J.J. Zielinski ${ }^{\mathrm{a}}$ and G. De Temmerman ${ }^{\mathrm{a}}$. \\ ${ }^{a}$ FOM Institute DIFFER, Association EUROATOM-FOM, Trilateral Euregio Cluster, P.O. Box \\ 1207, 3430 BE Nieuwegein, The Netherlands.
}

\begin{abstract}
.
Experiments were performed in the Pilot-PSI linear plasma device, to determine the sheath heat transmission coefficients in a high recycling regime under various conditions of density $\left(1-20 \times 10^{20} \mathrm{~m}^{-3}\right)$ and plasma composition $\left(\mathrm{H}_{2}, \mathrm{Ar}, \mathrm{N}_{2}\right)$ relevant for the ITER divertor plasma. The 2D surface temperature profile on a tungsten surface was measured with high spatial $(0.33 \mathrm{~mm})$ and temporal $(200 \mathrm{~Hz})$ resolution using an infrared camera. The target heat flux is calculated using a 2D axis-symmetric Ansys model, the heat transfer is determined from target calorimetry. The plasma parameters are measured with a high resolution Thomson scattering system located $17 \mathrm{~mm}$ away from the target surface. Radial profiles of the sheath heat transmission factors can thus be determined.

Preliminary results show that $\gamma$ varies between 4 and 40 depending on the plasma conditions and composition. The value derived from the heat flux calculated with ANSYS is significantly lower than theory predicts.
\end{abstract}

PSI-20 keywords: Tungsten, Divertor plasma, Thermography, Sheaths, Power deposition.

PACS: 52.40.Kh, 44.20.+b

Corresponding Author Address: FOM-Institute DIFFER, P.O. Box 1207, 3430 BE, Nieuwegein, The Netherlands

Corresponding Author e-mail: m.a.vandenberg@differ.nl

Presenting Author: M.A. van den Berg

Presenting Author e-mail: m.a.vandenberg@differ.nl 


\section{Introduction}

The study and quantification of heat loads to plasma-facing components both in terms of magnitude and spatial distribution is of high importance for the design of future devices as it impacts on the components lifetime. The heat flux to a surface can be experimentally determined from infrared thermography, thermocouples and Langmuir probes. Each of those methods presents inherent advantages and drawbacks. Infrared measurements provide excellent spatial and temporal resolutions but can be affected by plasma-induced surface modifications and reflections from surrounding surfaces [1]. The determination of heat fluxes from Langmuir probes relies on the sheath-heat transmission coefficient $(\gamma)$, which links the incoming heat flux and the plasma parameters at the surface [2]. $\gamma$ depends on the ratio of ion and electron temperature, secondary electron emission, ion mass, particle reflection coefficients, recombination energy, etc... Its determination for multi-species plasma such as the divertor plasma in ITER is hence not straightforward.

The Pilot-PSI device provides a unique advantage for studying plasma surface interaction; it produces plasma with ITER relevant densities and temperatures, and a well-diagnosed environment and access [3]. With Pilot-PSI the heat transmission factor can be determined and compared with the sheath theory. Experiments with different plasma conditions and gasses are used to expose tungsten targets.

\section{Experimental set up}

The Pilot-PSI device has been described in detail in $[3,4]$. The plasma source is a cascaded arc [5], which exhausts into the vessel along the magnetic field axis. To 
determine the dependence of the sheath heat transfer coefficient on the gas type, experiments are done with argon, nitrogen and hydrogen in high density plasmas with a Gaussian beam profile in both electron temperature and density. Radial profiles of plasma electron density $n_{e}$ and plasma electron temperature $T_{e}$ were measured with a Thomson scattering (TS) system $17 \mathrm{~mm}$ away from the surface of the target. A detailed description of the use of this diagnostic on Pilot-PSI can be found in [6]. This system can measure $n_{e}$ and $T_{e}$ profiles with a spatial resolution of $0.6 \mathrm{~mm}$ and an observational error of $3 \%$ and $6 \%$, respectively, at $n_{e}=4 \times 10^{19} \mathrm{~m}^{-3}$. These decrease to $1 \%$ and $2 \%$, respectively, at $n_{e}=$ $1 \times 10^{21} \mathrm{~m}^{-3}$. The flow and temperature of the target cooling water are measured, allowing the total power to the target to be determined by calorimetry. This system is calibrated with a heater with a known power output, the error is $10 \%$. A high speed IR camera (FLIR SC7500-MB) is used to measure the 2D target temperature profile during plasma exposure with a high spatial $(0.33 \mathrm{~mm})$ and temporal $(200 \mathrm{~Hz})$ resolution. The IR camera is calibrated with a black body and in situ cross checked against a multi-wavelength spectroscopic pyrometer (FAR SpectroPyrometer model FMPI) to account for the role of reflections inside the vessel and the transmission of the optical set up. The pyrometer measures a spectrum from 1000 to $1700 \mathrm{~nm}$, with a resolution of $1.56 \mathrm{~nm}$, corrects for the background and detector response and compares the corrected intensity with a black body radiator. After data analysis the output is the intensity per wavelength and the calculated emission spectra and temperature. With this, the emission and/or the transmission of the IR camera can be calibrated. In our case the emissivity (temperature and wavelength dependant) used for the temperature conversion is found in [7], and the transmission determined from the pyrometer is 0.9 . This reduces the error in the temperature to $2 \%$. 
Tungsten targets of $1 \mathrm{~mm}$ thick and $30 \mathrm{~mm}$ in diameter are used, clamped onto a water cooled copper heat sink, with grafoil ${ }^{\circledR}$ in between to ensure a good contact surface [8]. The target was kept at a floating potential. The targets are exposed to the plasma for 25 seconds with a magnetic field of $0.8 \mathrm{~T}$, TS is done after 20 seconds. In argon slightly higher source settings can be used; 225A through the arc with a gas flow of $2.4 \mathrm{slm}$. In hydrogen and nitrogen the source settings are 200 A with 2 slm.

\section{Results}

With the temperature from the IR camera and the calorimetry, the heat flux can be determined with Ansys. With the $T_{e}$ and $n_{e}$ from the TS system, the sheath heat transmission factor $\gamma$ to the surface can be estimated based on sheath theory [9]. The $\gamma$ from sheath theory can be compared to the $\gamma$ calculated with the heat flux from Ansys.

3.1 Sheath heat transmission factor from heat flux calculation with Ansys and TS

The temporal and spatial temperature from the IR camera is used as an input for a 2D axis symmetrical model in Ansys, consisting of a cylindrical tungsten target ((Ø $30 \times$ $1 \mathrm{~mm}$ ), a grafoil layer (Ø $22 \times 0.2 \mathrm{~mm})$ and a large copper heat sink $(\varnothing 45 \times 80 \mathrm{~mm}$ with a cutout in the middle for better water flow). For simplicity the clamping ring that ensures good contact between the target and the heat sink has not been taken into account, but the

pressure that is a result of the clamping ring is modeled as contact resistance. The boundary conditions are the temperature on the top surface, emission to the surrounding area, water cooling on the bottom of the copper heat sink and a contact resistance between the different materials. The contact resistance is pressure dependent, and can 
change with temperature due to different material expansion. The contact resistance results in an impaired heat flow through the model, and thus a different heat flux. This is modeled by two thermal conductance values, in the order of $6000-15000 \mathrm{~W} / \mathrm{m}^{2} \mathrm{~K}$ for both layers. Output of the simulation gives the time-resolved heat removed by the water and the time resolved heat flux $(q)$ per calculation cell, see fig. 1 . By changing the contact resistance between the layers, the heat removal through the water in Ansys is adjusted to match the calorimetric results. Although it is unknown what the ratio between the two contact resistances exactly is, the total resistance is what determines the heat flow through the whole model. The introduced error is therefore small, calculations show about $5 \%$.

The sheath heat transmission factor $\gamma$ can be calculated with $\gamma=q / k T_{e} \Gamma$, with $\Gamma$ the particle flux reaching the surface. $\Gamma$ can be calculated using the Bohm criterion for the sound speed at the sheath entrance; $c_{s}=\left[\left(k T_{e}+\gamma_{s} k T_{i}\right) / m_{i}\right]^{0.5}$ with $\gamma_{s}$ the specific heat ratio and $m_{i}$ the ion mass. In Pilot-PSI the $T_{i}$ is at least $T_{e}$ [10], for the following calculations $T_{e}$ $=T_{i}$ is used. As $T_{i}$ is at least $T_{e}$ a collisional adiabatic plasma is assumed. From fluid models the specific heat ratio is determined at 5/3 [9]. The particle flux than becomes $\Gamma=$ $0.5 n_{e} c_{s}$ so $\Gamma=0.5 n_{e}\left(8 / 3 k T_{e} / m_{i}\right)^{0.5}$.

\subsection{Sheath heat transmission factor based on sheath theory}

The $T_{e}$ and $n_{e}$ from TS for different gasses are fitted with a Gaussian function and depicted in fig. 2. For argon the source settings are higher, furthermore argon does not recombine into molecules, so the overall density is higher. The profile of argon is broader due to the higher mass. From these parameters plus the pre-sheath contributions $\left(\varepsilon_{\text {pres }}\right)$, 
the recombination of ions at the surface $\left(\chi_{i}\right)$, association to molecules $\left(\chi_{r}\right)$, secondary electron emission $\left(\delta_{e}\right)$ and reflection phenomena $\left(R_{i E}, R_{e E}, R_{i N}\right)$ [11] the sheath heat transmission factor $(\gamma)$ can be estimated according to Equation 1 [9].

$$
\begin{gathered}
\gamma \approx\left(\left[2.5 k T_{i}-0.5 k T_{e} \ln \left[\left(2 \pi \frac{m_{e}}{m_{i}}\right)\left(1+\frac{T_{i}}{T_{e}}\right)\left(1-\delta_{e}\right)^{-2}\right]\right]\left(1-R_{i E}\right)+\frac{2 k T_{e}}{1-\delta}\left(1-R_{e E}\right)+\right. \\
\left.\varepsilon_{\text {pre }}+\chi_{i}+\chi_{r}\left(1-R_{i N}\right)\right) / k T_{e}
\end{gathered}
$$

The pre-sheath contribution $\varepsilon_{p r e}=1 / 2 k T_{e}$, the energy and particle reflection coefficients are found in [11], but are for $10 \mathrm{eV}$, as no coefficients are available below that energy and the secondary electron emission yield $\delta_{e}$ for tungsten is computed with

$$
\delta_{e}=2.72^{2} \frac{E}{E_{\max }} e^{\left(-2\left(\frac{E}{E_{\max }}\right)^{1 / 2}\right)} \delta_{\max }
$$

with $E_{\max }=650 \mathrm{eV}, \delta_{\max }=1.4$ and $E=-k T_{e} 0.5 \ln \left[\left(2 \pi m_{e} / m_{i}\right)\left(1+T_{i} / T_{e}\right)\right]+0.7 k T_{e}$.

To estimate the importance of the different factors on the $\gamma$, the different components are listed in table 1, for the maximum electron temperature, i.e. in the middle of the plasma beam. For comparison the values are divided by the $k T_{e}$, so the values can be added up to form the sheath heat transmission.

Table 1. The different components of the sheath heat transmission factor for 3 different gasses.

\begin{tabular}{|c|c|c|c|}
\hline & Argon & Hydrogen & Nitrogen \\
\hline Pre sheath voltage & 0.5 & 0.5 & 0.5 \\
\hline Sheath voltage & 5.1 & 2.1 & 3.4 \\
\hline Electron reflection & 1.8 & 1.7 & 1.7 \\
\hline$\chi_{\mathrm{i}}$ & 8.9 & 7.0 & 11.1 \\
\hline$\chi_{\mathrm{r}}$ & 0 & 0.27 & 0.86 \\
\hline$\gamma$ & 16.3 & 11.6 & 17.7 \\
\hline
\end{tabular}


This table shows that the recombination of ions at the surface is the most important contribution, for all gasses. In figure 3 the $\gamma$ is depicted versus the electron temperature. This graph shows that for small $T_{e}$ the $\gamma$ varies strongly, so a small variation in $T_{e}$ results in a large variation in $\gamma$.

\subsection{Comparison and discussion}

A comparison between the sheath heat transmission factors based on heat flux calculations determined from thermography and those expected from equation 1 is shown in figure 4. For argon the qualitative shape is very reasonable, but quantitatively the $\gamma$ calculated from sheath theory is a factor 5 higher. For hydrogen the qualitative shape is very reasonable in the middle, although at the edge the shape begins to deviate. In the middle the $\gamma$ calculated from sheath theory is a factor 4 higher. For nitrogen the qualitative shape does not agree very well, although the qualitative value agrees the best for the three gasses; in the middle the $\gamma$ calculated from sheath theory is a factor 2 smaller.

In this low energy, high flux regime some effects must be taken into account; a plasma flow with the possibility of a density gradient at the TS and a pre sheath that is at least affected with collisions with neutrals. Furthermore these fluxes could mean theory of sheath limited regime is not applicable, but is more likely a high recycling regime. To see if reevaluation of the assumptions made could explain the difference between the $\gamma$ 's, they are listed below.

There are almost no reflection coefficients available for energies lower than 10 $\mathrm{eV}$. With equation 2 the $\delta_{e}$ for all gasses used, are in the order of 0.1 or lower. Gunn [12] 
shows that in Tore Supra measured secondary electron emission yields corresponds much better for low $T_{e}$ if backscattering is taken into account, which results in $\delta_{e}$ values around 0.8. Although this is obtained for hydrogen implanted graphite, the same effect could hold for tungsten. If the backscattering of electrons is taken into account in the secondary emission yield, the sheath voltage drops, but the electron reflection energy grows faster, so in our case, the $\gamma$ based on sheath theory increases by a factor of 0.5 .

As the electron temperatures are low, the re-ionization is negligible, so the presheath is in the order of the mean free path for ion-neutral momentum exchange [13], for these conditions $\sim 1 \mathrm{~cm}$. Although the TS is $17 \mathrm{~mm}$ away from the target, there could still be a density gradient. In similar hydrogen conditions, Shumack [13] has done measurements in the pre-sheath and found that the density drop can be more than the normal factor 2, due to the friction between ions and neutrals, thereby decreasing the particle flux up to a factor of 2, and thus increasing the $\gamma$ derived from Ansys more than factor 2 .

Futch [14] compares experimental data from DIII-D (deuterium plasma with $T_{e}=$ $T_{i}=20 \mathrm{eV}$ ) with theory including momentum-changing collisions of the plasma ions with neutral gas. With growing neutral pressure, the $\gamma$ from sheath theory can be reduced more than factor 2.

The assumption in the specific heat ratio $\gamma_{s}$. The $\gamma_{s}$ can range from 1 to 3 , so the subsequent error in the flux is $25 \%$.

The measurements errors in the $\gamma$ consists of the error in the calorimetry (10\%), the error in the temperature readout (2\%), the uncertainty in the Ansys calculation (5\%), and the error in the TS (3\%). The total measurement error amounts to $20 \%$, which is in 
the same order of measurements done with the total saturation current $\left(\mathrm{I}_{\text {sat }}\right)$ in Pilot-Psi [15], where ion flux from TS and from $\mathrm{I}_{\text {sat }}$ was compared and found to be 30\%.

\section{Conclusions}

During plasma exposures of tungsten targets to ITER divertor relevant plasmas (high $\mathrm{n}_{\mathrm{e}}$, low $\mathrm{T}_{\mathrm{e}}$ ) in Pilot-PSI, experimental and theoretical heat transfer coefficients are determined for different gasses. A comparison between the $\gamma$ determined from the heat flux calculated with Ansys divided by the electron temperature and particle flux and the $\gamma$ estimated from sheath theory are made for argon, hydrogen and nitrogen. These results are a first step towards a better understanding of the sheath heat transmission factors in a high recycling regime.

In all cases, the $\gamma$ derived from the heat flux calculated with Ansys is significantly lower than sheath limited regime predicts.

If the plasma flow in Pilot-PSI and the neutral pressure are taken into account the differences in $\gamma$ can become a factor of 4 less. This could explain the deviation in hydrogen and nitrogen, and a large part of the difference in Argon.

If the enhanced backscattering of electrons is taken into account the difference in $\gamma$ becomes larger.

\section{Acknowledgement}

This work was supported by the European Communities under the contract of Association between EURATOM/FOM and carried out within the framework of the 
European Fusion Program with financial support from NWO. The views and opinions expressed herein do not necessarily reflect those of the European Commission. 


\section{References}

[1] G. De Temmerman et al, Plasma Phys. Control. Fusion, 52 (2010) 095005

[2] R.A. Pitts et al., Plasma Phys. Control. Fusion 47 (2005) B303-B305.

[3] J. Westerhout et al., Phys. Scr. T128 (2007) 18-22.

[4] G. J. van Rooij et al., Appl. Phys. Lett. 90 (2007) 121501.

[5] W. A. J. Vijvers et al., Phys. Plasmas 15 (2008) 093507.

[6] H. J. van der Meiden et al., Rev. Sci. Instr. 79 (2008) 013505.

[7] D.R. Lide and H.P.R. Frederikse, CRC handbook of Chemistry and Physics 75th Edition, CRC Press (1994-1995)

[8] M.A. van den Berg et al., Fus Eng Des 86 (2011) 1745-1748

[9] P.C. Stangeby, The plasma boundary of magnetic fusion devices, IOP (2000)

[10] A.E. Shumack et al., Phys Rev E 78, (2008) 046405

[11] W. Eckstein, Calculated sputtering, refection and range values, IPP-Report IPP 9/132 (2002)[12] J.P.Gunn, Plasma Phys. Control. Fusion 54 (2012) 085007

[13] A.E. Shumack, PhD thesis, The influence of electric fields and neutral particles on the plasma sheath at ITER divertor conditions (2011)

[14] A.H. Futch et al., Journal of Nuclear Materials 196-198, (1992) 860-864

[15] J. Westerhout, PhD thesis, Carbon chemical erosion in high flux and low temperature hydrogen plasma (2010) 


\section{Figure captions:}

Fig. 1. The heat flux calculated with Ansys from the IR camera temperature and the calorimetry. The upper graph shows the profile at 20 seconds, the bottom graph shows the maximum heat flux plotted versus time.

Fig 2. The upper graph shows the electron density and the Gauss fits, taken from Thomson scattering measurements at 20 seconds, the lower graph shows the electron temperature and the gauss fits.

Fig. 3. Sheath heat transmission factor versus electron temperature for different gasses calculated with sheath theory based on Thomson scattering measurements.

Fig. 4. Sheath heat transmission factors based on heat flux calculations with Ansys, using IR and calorimetry, divided by particle flux from TS and based on Thomson scattering measurements and sheath theory versus the radius in Pilot-PSI for different gasses; argon, hydrogen and nitrogen. 

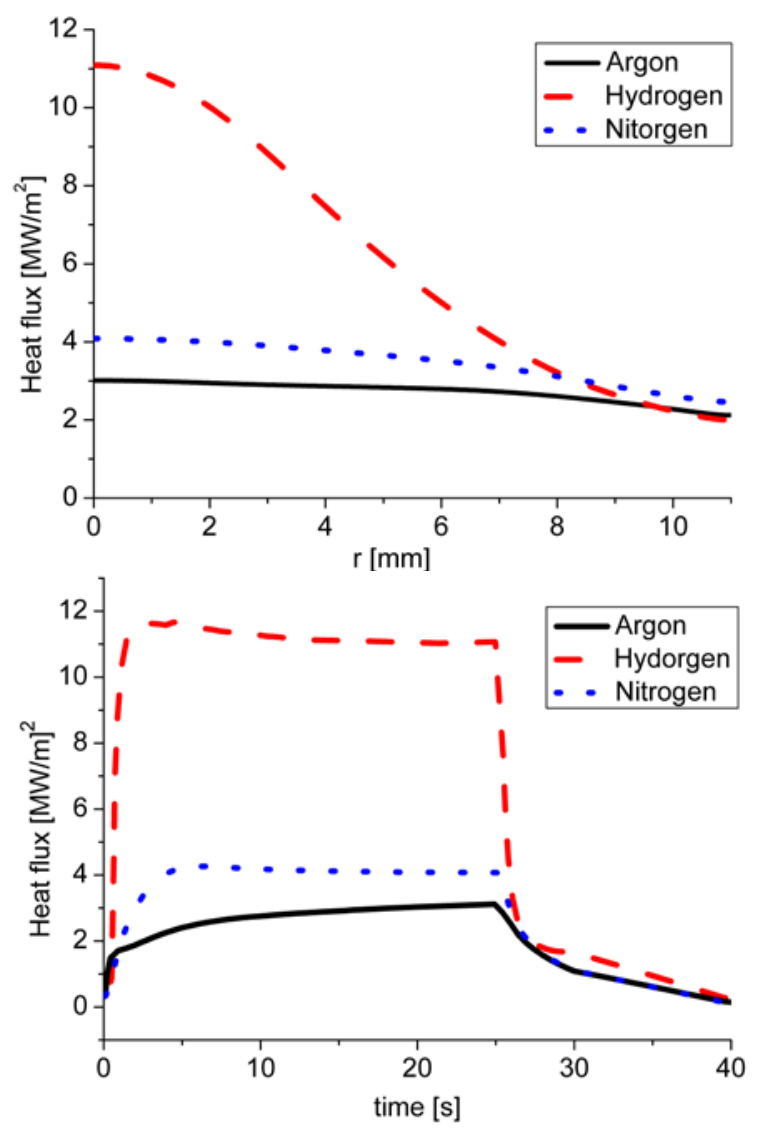

Fig. 1. The heat flux calculated with Ansys from the IR camera temperature and the calorimetry. The upper graph shows the profile at 20 seconds, the bottom graph shows the maximum heat flux plotted versus time. 

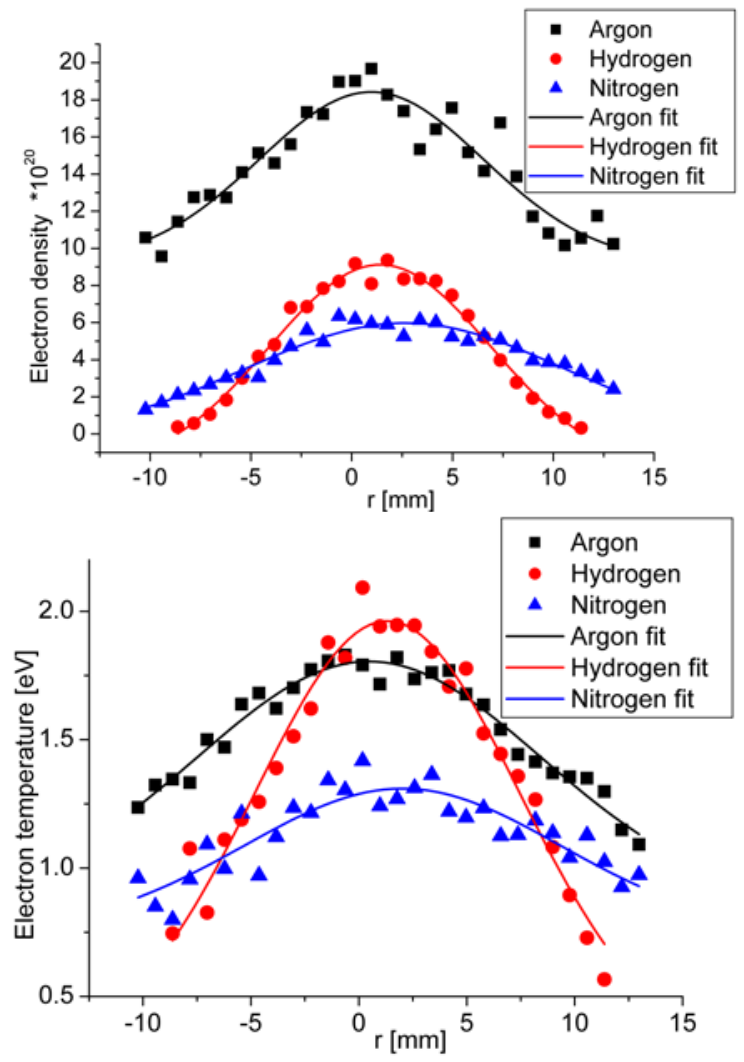

Fig 2. The upper graph shows the electron density and the Gauss fits, taken from Thomson scattering measurements at 20 seconds, the lower graph shows the electron temperature and the gauss fits. 


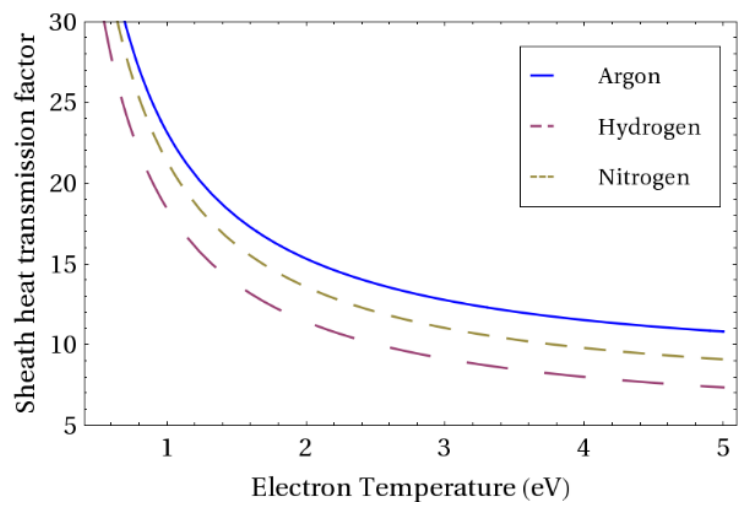

Fig. 3. Sheath heat transmission factor versus electron temperature for different gasses calculated with sheath theory based on Thomson scattering measurements. 



Fig. 4. Sheath heat transmission factors based on heat flux calculations with Ansys, using IR and calorimetry, divided by particle flux from TS and based on Thomson scattering measurements and sheath theory versus the radius in Pilot-PSI for different gasses; argon, hydrogen and nitrogen. 